\title{
Disposable integrated microfluidic biochip for blood typing by plastic microinjection moulding $\dagger$
}

\author{
Dong Sung Kim, ${ }^{a b}$ Se Hwan Lee, ${ }^{c}$ Chong H. Ahn, ${ }^{c}$ Jae Y. Lee ${ }^{d}$ and Tai Hun Kwon*ab \\ Received 23rd November 2005, Accepted 24th March 2006 \\ First published as an Advance Article on the web 4th April 2006 \\ DOI: 10.1039/b516495h
}

Blood typing is the most important test for both transfusion recipients and blood donors. In this paper, a low cost disposable blood typing integrated microfluidic biochip has been designed, fabricated and characterized. In the biochip, flow splitting microchannels, chaotic micromixers, reaction microchambers and detection microfilters are fully integrated. The loaded sample blood can be divided by 2 or 4 equal volumes through the flow splitting microchannel so that one can perform 2 or 4 blood agglutination tests in parallel. For the purpose of obtaining efficient reaction of agglutinogens on red blood cells (RBCs) and agglutinins in serum, we incorporated a serpentine laminating micromixer into the biochip, which combines two chaotic mixing mechanisms of splitting/recombination and chaotic advection. Relatively large area reaction microchambers were also introduced for the sake of keeping the mixture of the sample blood and serum during the reaction time before filtering. The gradually decreasing multi-step detection microfilters were designed in order to effectively filter the reacted agglutinated RBCs, which show the corresponding blood group. To achieve the cost-effectiveness of the microfluidic biochip for disposability, the biochip was realized by the microinjection moulding of COC (cyclic olefin copolymer) and thermal bonding of two injection moulded COC substrates in mass production with a total fabrication time of less than $20 \mathrm{~min}$. Mould inserts of the biochip for the microinjection moulding were fabricated by SU-8 photolithography and the subsequent nickel electroplating process. Human blood groups of A, B and AB have been successfully determined with the naked eye, with $3 \mu$ of the whole sample bloods, by means of the fabricated biochip within $3 \mathrm{~min}$.

\section{Introduction}

Blood typing is the most important test performed for blood donors and recipients whenever transfusion is required. The critical importance of blood typing, especially for ABO blood group, stems from the fact that the all agglutinins of the $\mathrm{ABO}$ system fix complement and they could even cause intravascular hemolysis of incompatible red cells. Therefore, a mistyping of the $\mathrm{ABO}$ blood group of recipients or donors brings about an undesirable tragic result. The blood group is generally determined by observing agglutination results of the red blood cells (RBCs) reacting with the corresponding blood serum. The agglutination of RBCs shows the presence of antigens (agglutinogens) on the RBCs corresponding to antibodies (agglutinins) in the serum. The determination of the ABO

\footnotetext{
${ }^{a}$ Department of Mechanical Engineering, Pohang University of Science and Technology (POSTECH), San 31 Hyoja-dong Nam-gu, Pohang,

Kyungbuk 790-784, Korea. E-mail: thkwon@postech.ac.kr;

Fax: 8254 2795899; Tel: 82542792175

${ }^{b}$ Research Center for Micro Mechanical Systems, Pohang University of Science and Technology (POSTECH), San 31 Hyoja-dong Nam-gu,

Pohang, Kyungbuk 790-784, Korea

${ }^{c}$ MicroSystems and BioMEMS Laboratory, Department of Electrical \& Computer Engineering and Computer Science, University of Cincinnati, P. O. Box 210030, Cincinnati, OH 45221-0030, USA

${ }^{d}$ College of Medicine, Ohio State University, Columbus, OH 45221,

USA

$\uparrow$ Electronic supplementary information (ESI) available: supplemen-
} tary figures. See DOI: $10.1039 / \mathrm{b} 516495 \mathrm{~h}$ blood group is possible by two different typing procedures: red cell typing (also called forward grouping) and serum typing (reverse grouping). The purpose of performing both cell and serum typing is to confirm the agglutination results of each other. From these typing procedures, one of the blood groups of $\mathrm{A}, \mathrm{B}, \mathrm{AB}$ and $\mathrm{O}$ can be accurately assigned to the test blood sample because red cells agglutinate each other when they react with the corresponding blood grouping serum, Anti-A or Anti-B, as mentioned above. Blood typing sera, Anti-A, Anti-B and Anti-AB, are obtained from the sera of blood group $\mathrm{B}$ persons, $\mathrm{A}$ persons and $\mathrm{O}$ persons, respectively. It should be mentioned that Anti-AB is not a simple mixture of Anti-A and Anti-B but a particular serum of blood group $\mathrm{O}$ persons, which can be applied to determining weak antigens of red cells, in particular weak-A antigens (e.g., $\mathrm{A}_{2}, \mathrm{~A}_{3}, \mathrm{~A}_{\text {int }}$, etc $) .{ }^{1}$ It should also be noted that there are subgroups of $\mathrm{A}$ and $\mathrm{B}$, which are grouped as weak-A and weak-B (e.g., $\mathrm{B}_{3}, \mathrm{~B}_{\text {int }}$, etc.), respectively. Since they result in weak agglutination in red cell typing, the test blood sample could be even mistyped as group O. As mentioned above, the mismatched transfusion brings about intravascular hemolysis of the patient. To prevent this mistyping from happening, special attention is required for doing red cell typing.

Among many blood typing methods, a plate method, ${ }^{1,2}$ a gel card method $^{3,4}$ and an automated blood bank test system ${ }^{5,6}$ have been regarded as the most representative blood typing methods. The plate method is relatively simple but tedious due 
to its whole manual process. Furthermore, it may result in undesirable mistyping of blood group of a sample blood due to poor reliability and user performance. The gel card method is regarded as a semi-automatic and relatively accurate test method. However, the test kit is expensive and it requires a practised hand and extra equipment, such as a centrifuge. The blood bank system offers fully automated blood typing of numerous bloods. This system, however, is very expensive and huge so that only major medical centers or blood banks can maintain it. Recently, Lee et al. ${ }^{7}$ presented a miniaturized blood typing system fabricated by the micro-stereolithography technology, which assembled flow splitting channels, chaotic micromixers ${ }^{8}$ and reaction chambers. They used $20 \mu \mathrm{l}$ of a sample blood per test line to determine the blood group and showed that an efficient micromixer should be required to get reliable agglutination reaction in microchannels. However, their system has the limitations of disposability and portability due to the high fabrication cost and requirement of extra detection equipment to observe agglutinated red cells in the reaction chambers. ${ }^{7}$

Therefore, the previous blood typing methods including the plate, gel card, automated system and miniaturized system explained above have a limitation for emergency blood typing due to the requirement of extra-large scale equipment and much testing time for blood sedimentation. In other words, they are not suitable for point-of-care monitoring and treatment, especially for the emergency case. Furthermore, the previous methods generally require a blood volume of more than $20 \mu \mathrm{l} .^{5}$ If the repeated collection of blood from patients is required, withdrawing blood becomes a critical problem, in particular for infants though the test blood volume is relatively small.

In this regard, we propose a low cost, lab-on-a-chip for blood typing, integrated microfluidic biochip of compact size, which requires a very small sample blood volume of the order of $1 \mu \mathrm{l}$. In the following sections we introduce the design and operation process of the microfluidic biochip and components integrated in the biochip: flow splitting microchannels, chaotic micromixers, ${ }^{9}$ reaction microchambers and detection microfilters. As the cost-effective mass fabrication method of the biochip, a microinjection moulding process with electroplated nickel mould inserts and a subsequent thermal bonding process are applied. Then, we present successful blood typing results of blood groups of $\mathrm{A}, \mathrm{B}$ and $\mathrm{AB}$ by means of the fabricated biochip.

\section{Theories and design of biochip}

\section{Determination of $\mathrm{ABO}$ blood group}

The ABO blood group can be determined by reacting sample RBCs with the corresponding blood grouping serum, e.g., Anti-A and Anti-B. For instance, RBCs of blood group A are agglutinated by Anti-A. Table 1 lists the determination method of $\mathrm{ABO}$ blood group.

In general, both red cell typing and serum typing are carried out for the accurate determination of blood group in order to compare the agglutination results of the other test. Red cell typing could have seven test channels, which contain sample RBCs with standard Anti-A, Anti-B, Anti-AB,
Table 1 Determination of ABO blood group from reaction results

\begin{tabular}{lll}
\hline \multicolumn{2}{l}{ Reaction result of sample red blood cells with- } & ABO blood \\
Anti-A & group typing \\
\hline$(-)$ & Anti-B & O \\
$(+)$ & $(-)$ & A \\
$(-)$ & $(-)$ & B \\
$(+)$ & $(+)$ & AB
\end{tabular}

${ }^{a}(+)$ and $(-)$ mean the existence and absence of agglutinated red blood cells (filtered cells in this case, which will be explained later), respectively.

Anti-A $\mathrm{A}_{1}$, Anti-H, Anti-D and negative control, to determine blood groups of $\mathrm{A}, \mathrm{B}, \mathrm{AB}, \mathrm{O}, \mathrm{Rh}$ (D) and also weak-A. Anti- $\mathrm{A}_{1}$ and Anti-H are used for determining weak blood subgroups and negative control is required to check the selfagglutination of the sample blood. For serum typing, three test channels, which contain sample serum with standard red cell-A, cell-B and cell-O, could be applied to confirm the test result of the red cell typing.

\section{Overall design and operation process of biochip}

Fig. 1 shows a schematic diagram of one blood typing unit in a microfluidic biochip designed in this study. It might be mentioned that one biochip, in this study, was designed to have two blood typing units and each blood typing unit has four test lines. The designed blood typing microfluidic biochip fully integrates flow splitting microchannels, chaotic micromixers, reaction microchambers and detection microfilters, as indicated in Fig. 1. The present biochip is composed of two substrate layers to increase the storage volume capacity of the reaction chamber and to consider the design of a serpentine laminating micromixer (SLM $)^{9}$ as a chaotic micromixer. The thickness of all the microfluidic channels in each substrate is designed to be $60 \mu \mathrm{m}$.

A blood typing process by means of the biochip in this study runs as follows. The constant volume (about $1-1.5 \mu \mathrm{l}$ per test line) of a sample blood (RBCs) and the standard sera are loaded into the corresponding inlets, as indicated in Fig. 1(a) (process step (i) in Fig. 1(b)). The loaded sample blood and standard sera are injected into the biochip through the flow splitting microchannels via a syringe pump, for example. In this case, the stream of the sample blood splits into and is guided to 2 or 4 separate microchannels (process step (ii)). Both the divided blood stream and the corresponding serum stream are introduced into each connected chaotic micromixer, SLM, to be efficiently mixed with each other in order to induce the effective agglutination of RBCs with the corresponding antibodies (process step (iii)). The mixtures of blood and serum streams are then led to each reaction microchamber. The reaction microchamber keeps the mixture during the appropriate reaction time about $1-3 \mathrm{~min}$ for agglutination of RBCs. It should be mentioned that, in the present study, we stopped the syringe pump at this process step to eliminate the driving pressure applied to the biochip, thereby keeping the mixtures inside the reaction microchambers during the reaction time (process step (iv)). The reacted (or non-reacted) mixture of blood RBCs and serum passes through each detection microfilter and finally flows out from each outlet (process 

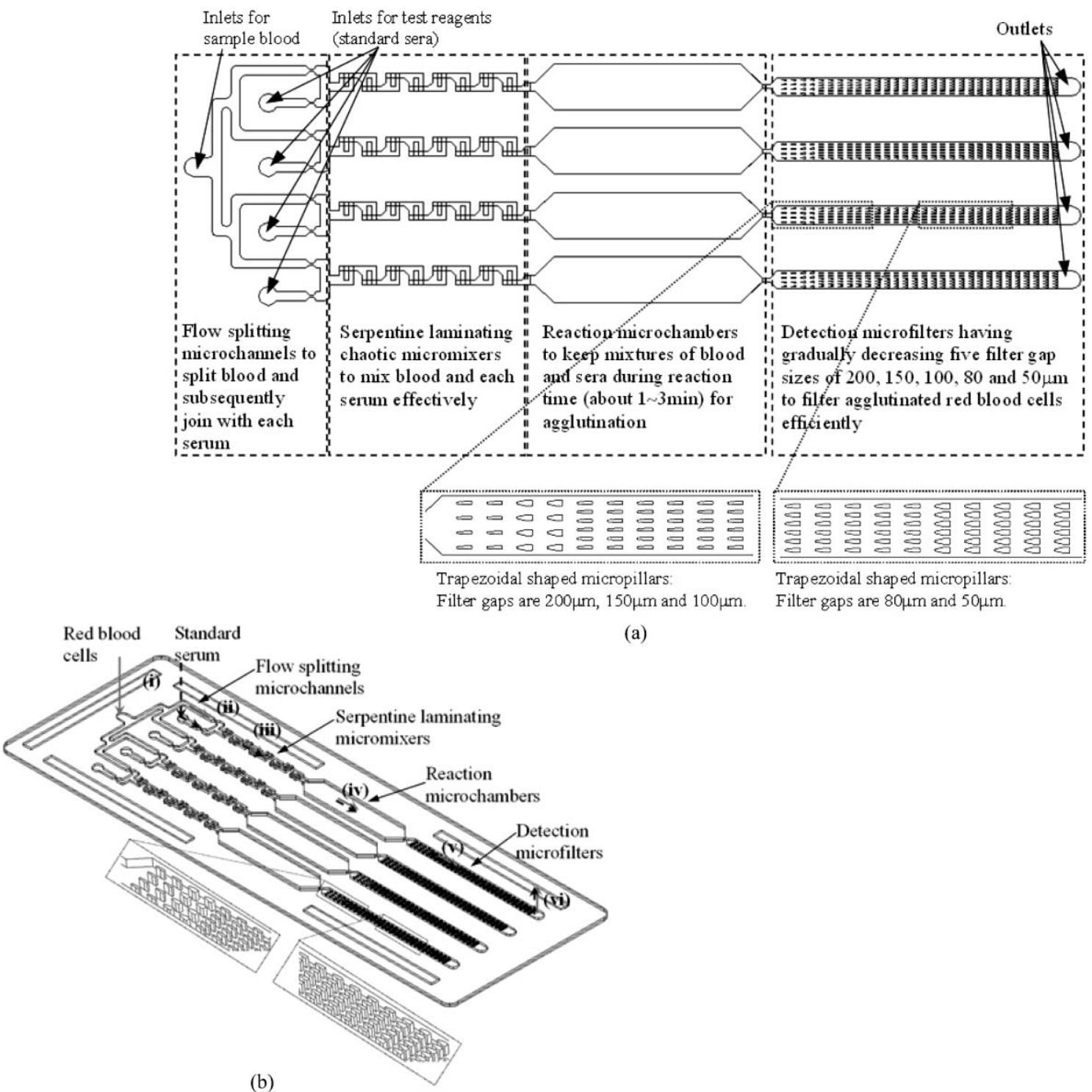

(a)

Fig. 1 Schematic diagram of one blood typing unit of a microfluidic biochip in which flow splitting microchannels, chaotic micromixers, reaction microchambers and detection microfilters are fully integrated. The reaction microchamber can store about $3 \mu$ of a mixture of blood and serum. The agglutinated red blood cells formed by antigen-antibody reaction between red blood cells and test serum can be effectively observed by the efficient detection microfilters (insets are enlarged views of the microfilter): (a) top view; and (b) three-dimensional view.

steps (v) and (vi)). The large size reacted agglutinated cells are effectively filtered inside each detection microfilter, whereas non-reacted RBCs easily pass the detection microfilters. Therefore, one can observe the agglutination results by the naked eye without any extra large-scale equipment such as a microscope, and thus one can easily determine the blood group of the test blood sample by means of the biochip alone.

\section{Flow splitting microchannel}

In the present biochip, the loaded sample blood is divided into 2 or 4 equal volumes through the flow splitting microchannels, as explained in Fig. 1. The 4 divided streams of the sample blood and sera injected are finally guided to the front of the subsequent micromixers by means of the flow splitting microchannels. The width of the main channel was designed to be $500 \mu \mathrm{m}$ in this study, and thereby all of the microchannels have the same width and thickness.

It might be noted that one can perform 2 or 4 blood agglutination tests in parallel with the present design. For the case of 2 blood agglutination tests (e.g., with Anti-A and Anti-B, that is a simple ABO blood typing), one can utilize two of four test lines in the one blood typing unit in Fig. 1 by opening two inlets of sera and the corresponding two outlets (of course, also opening blood inlet). For the 4 blood agglutination tests (e.g., with Anti-A, Anti-B, Anti-D and Anti- $A_{1}$ ), all the four test lines are utilized by opening all inlets and outlets. Finally, it might be mentioned that when there exists any need to design multiple flow splitting microchannels having odd splitting channels or different splitting volumes, one can utilize the microchannel design equations suggested in ref. 10 to achieve that. 


\section{Serpentine laminating micromixer}

As was mentioned above, for accurate blood typing efficient mixing of RBCs and serum is definitely desired. ${ }^{1,7}$ Because of the low Reynolds number $(R e)$ of the microchannel flow in the biochip, however, it is impossible to expect turbulent mixing of two fluid flows. In this regard, we have newly developed an efficient chaotic micromixer having a simple geometry, named serpentine laminating micromixer (SLM), as indicated in Fig. $1,{ }^{9}$ to effectively incorporate into the present disposable microfluidic biochip. The SLM combines two general chaotic mixing mechanisms of splitting/recombination and chaotic advection, thereby resulting in the higher level of chaotic mixing in the microchannel. In the present SLM, eight "F"-shaped mixing units are successively arranged in two layers. The main channel width of the F-shaped mixing unit is $250 \mu \mathrm{m}$.

The SLM has a great advantage of mass producible simple geometry while maintaining the higher level of chaotic mixing over the wide range of Re. Furthermore, with the SLM, one could effectively reduce the chances of non-specific adsorption of proteins (antibodies in this study) to the channel surface by decreasing contact area between the surface of the microchannel and stream.

Two sample RBCs and standard serum streams injected from the connected flow splitting microchannels are efficiently mixed up through the SLM. Each mixture of RBCs and serum is then led to the subsequent reaction microchamber. For the detailed mixing mechanism and characterization of the SLM, one may refer to ref. 9.

\section{Reaction microchamber}

In the present blood typing method with the microfluidic biochip, the blood group of the sample blood is determined by observing the agglutination of RBCs by antibodies in serum. In this regard, the observation of agglutination (in this case, filtering) should be carried out after the proper reaction time of agglutination of RBCs. Therefore, the mixture of RBCs and serum should be kept during the reaction time of about 1-3 min before filtering for detection.

Fig. 1 schematically shows the reaction microchamber between the SLM and the detection microfilter. The width and total thickness of the reaction chamber was designed to become $2500 \mu \mathrm{m}$ and $120 \mu \mathrm{m}$ (two layers), respectively. As was mentioned before, the reaction microchamber is patterned both top and bottom substrates for the purpose of increasing the storage volume capacity of the chamber, so that the reaction microchamber can contain about $3.3 \mu$ of mixture of blood and serum.

After the prescribed reaction time, reacted (or non-reacted) mixtures of blood and serum are injected into the connected detection microfilters for observation of agglutination.

\section{Detection microfilter}

In the present study, we have designed a gradually decreasing multi-step detection microfilter to observe agglutinated red cells with the naked eye, without large optical detection systems, for the purpose of achieving the portability as well as the disposability of the biochip. The typical size of a red blood cell is $7.6 \mu \mathrm{m}$ in diameter and $2.3 \mu \mathrm{m}$ in thickness. Interestingly, the antigen-antibody reaction between $\mathrm{RBCs}$ and the corresponding serum could form large size agglutinated RBCs even greater than $100 \mu \mathrm{m}$. One could determine the size of the filter gap by considering the usual size of agglutinated red cells.

Insets of Fig. 1 schematically show the detection microfilters designed in this study. Various trapezoidal planar shaped micropillars that make the filters are periodically arranged at the regular interval of $500 \mu \mathrm{m}$ in the downchannel direction. The trapezoidal shape of the micropillars makes a wide inlet and a narrow outlet of the filter gaps. This wide inlet guides the injected agglutinated or non-agglutinated RBCs to be easily flowed into the narrow outlet so that it enhances the efficiency of the microfilter. The detection microfilter was designed to have five gradually decreasing outlet filter gaps, i.e., the minimum distances between the adjacent micropillars in the transverse direction, along the downstream direction: $200 \mu \mathrm{m}$ ( 2 columns), $150 \mu \mathrm{m}$ ( 2 columns), $100 \mu \mathrm{m}$ ( 6 columns), $80 \mu \mathrm{m}$ (10 columns) and $50 \mu \mathrm{m}$ (10 columns) (see electronic supplementary information (ESI) for detailed dimensions of the microfilter $\dagger$ ). It might be mentioned that we determined the minimum size of the micropillar features and the filter gaps as $50 \mu \mathrm{m}$, taking into account processing conditions of photolithography and microinjection moulding processes, which are the fabrication method of the present disposable microfluidic biochip. With the present design of the microfilter, normal RBCs can easily pass the filter gaps without any interference, whereas the large size agglutinated RBCs of which the size is greater than $50 \mu \mathrm{m}$ could not pass the filter gaps and thereby are filtered during the flow through the microfilter.

\section{Fabrication}

\section{Fabrication of mould insert}

To realize the integrated microfluidic biochip in mass production is one of the principal objectives of this work. The biochip was realized by means of a microinjection moulding process, which is one of the most promising mass production methods of polymer materials. In this regard, it is first required to fabricate mould inserts for the replication of the substrates of the biochip with the microinjection moulding. Typical materials used in a micromachining process, such as silicon, photoresists or polymeric materials, cannot be directly used as a mould or a mould insert due to their weak strength or thermal properties in the microinjection moulding process. It is desirable to use metallic materials which have appropriate mechanical and thermal properties to endure both a high pressure and a large temperature variation during the replication process. Therefore, a metallic mould insert is used rather than the silicon wafer or polymer substrate itself for mass production with such replication technologies. Otherwise, special techniques should be adopted as a replication method, e.g., casting of PDMS [poly(dimethylsiloxane)] elastomer. ${ }^{11}$ In this study, nickel mould inserts were fabricated for the biochip by a nickel electroplating process with SU-8 photoresist patterns on nickel substrates. 


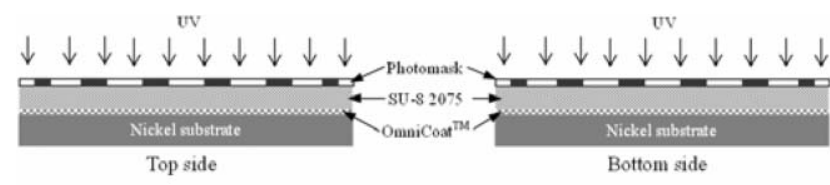

(a)
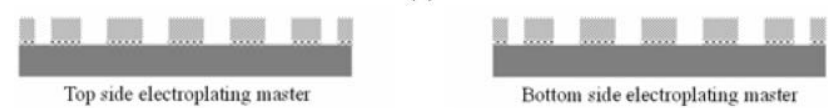

(b)

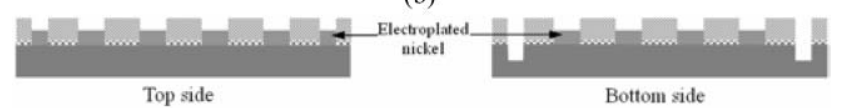

(c)
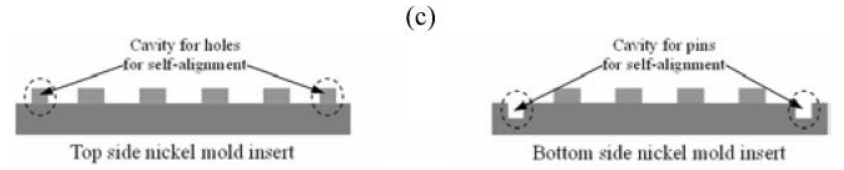

(d)

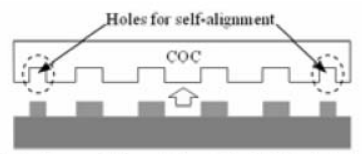

Injection molding of top side COC substrate

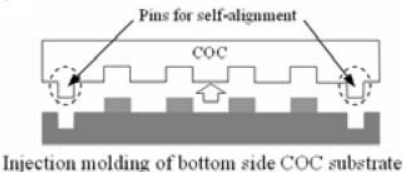

(e)

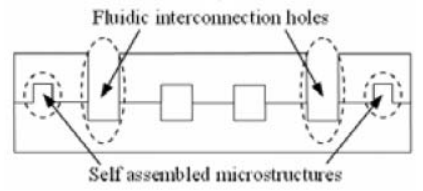

(f)

Fig. 2 Fabrication process of the blood typing integrated microfluidic biochip: (a) UV photolithography, (b) SU-8 and OmniCoat $(\mathbb{M})$ layers patterned on the nickel substrates as masters for electroplating, (c) electroplated nickel in the masters, (d) nickel mould inserts after removing SU-8 and OmniCoat $(\mathrm{MM})$ layers, (e) injection moulding of COC substrates and (f) final microfluidic biochip after thermal bonding.

Fig. 2 illustrates the schematic diagram of the whole fabrication process of the blood typing integrated microfluidic biochip. The fabrication process of the mould inserts is as follows (Fig. 2(a)-2(d)). First of all, two chrome photomasks corresponding to the top and bottom layers of the biochip were fabricated based on the design shown in Fig. 1. Two nickel substrates, of which the diameter and thickness are 3 inch and $1.5 \mathrm{~mm}$, respectively, were prepared as the mould inserts for the microinjection moulding process. The nickel disks were cleaned and then dried in a $120^{\circ} \mathrm{C}$ oven for about $2 \mathrm{~h}$ before the spin coating process.

In order to improve the adhesion between the nickel surface and the thick negative photoresist of SU-8, first an adhesion promoter, OmniCoat $\mathrm{IM}$ (Microchem Corp., MA, USA), was spin coated over the nickel substrates at a speed of $3000 \mathrm{rpm}$ for $30 \mathrm{~s}$ and baked on the hot plate at $200{ }^{\circ} \mathrm{C}$ for $1 \mathrm{~min}$. The SU-8 2075 (Microchem Corp., MA, USA) was spin coated on the baked thin OmniCoat $\mathrm{TM}$ layer at a speed of $950 \mathrm{rpm}$ for 45 s. After spin coating, a soft baking process was carefully performed at $65{ }^{\circ} \mathrm{C}$ for $10 \mathrm{~min}$ and at $95{ }^{\circ} \mathrm{C}$ for $1 \mathrm{~h}$ and $45 \mathrm{~min}$. Then, UV light was exposed to the soft baked SU-8 and OmniCoat(IM) layers on the nickel substrates with a total dose

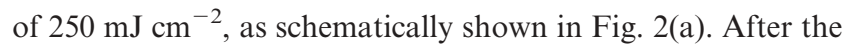
UV exposure, a post exposure baking (PEB) process was carried out at $65{ }^{\circ} \mathrm{C}$ for $10 \mathrm{~min}$ and at $95{ }^{\circ} \mathrm{C}$ for $10 \mathrm{~min}$. Then, both SU-8 and OmniCoat $(\mathrm{MM}$ layers were developed by immersing the nickel substrates into both a SU-8 developer and an OmniCoat $\mathrm{IM}$ ) developer (Microposit MF 319) to obtain masters for the electroplating process as depicted in Fig. 2(b).

As a final step of the mould insert fabrication, the electroplating process was carried out in a nickel electroplating bath with the prepared electroplating masters at a current density of $10 \mathrm{~mA} \mathrm{~cm}^{-2}$ for $6 \mathrm{~h}$ (Fig. 2(c)). Finally, two nickel mould inserts, which contain $60 \mu \mathrm{m}$ thick electroplated nickel microstructures on the nickel substrates, of the microfluidic biochip for the microinjection moulding were achieved after stripping the residual SU-8 2075 and OmniCoat $\mathbb{T M}$ layers by immersing them in a stripper (Remover PG), as schematically shown in Fig. 2(d). It should be noted here that in order to realize the precise alignment of two polymer layers of the biochip during the bonding stage, the cavities of pairs of pins and holes, the complementary self-alignment microstructures, were patterned on two nickel mould inserts as indicated in Fig. 2(d). ${ }^{12}$ One may refer to ref. 12 for the detailed process of this self-assembly technique.

Figs. 3 and 4 show the photographs and representative titled SEM (scanning electron microscope) pictures of the two fabricated nickel mould inserts. It might be noted that one can clearly observe the minimal surface roughness of the

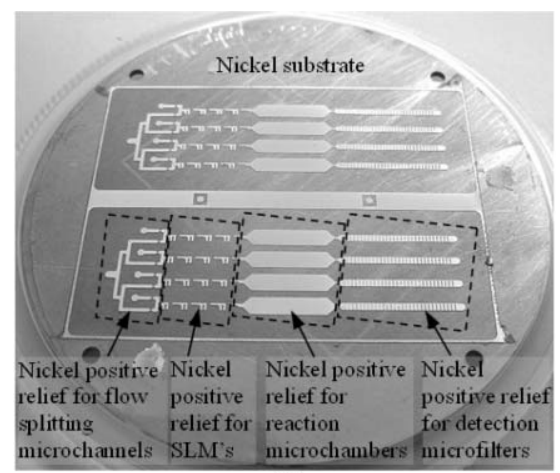

(a)

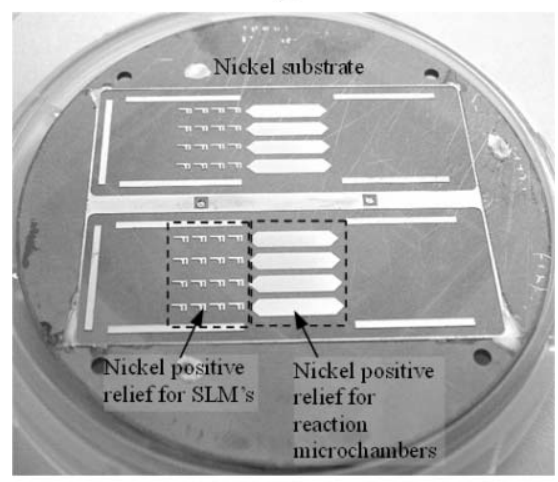

(b)

Fig. 3 Mould inserts of the blood typing integrated microfluidic biochip for the microinjection moulding process, which contain $60 \mu \mathrm{m}$ thick electroplated nickel positive relief on the nickel substrate: (a) top side; and (b) bottom side. 


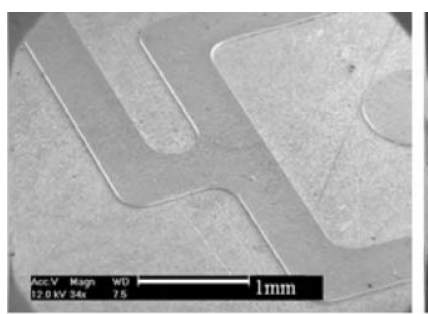

(a)

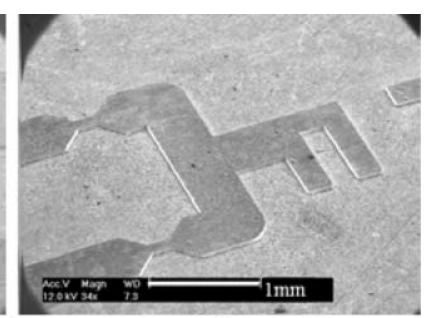

(b)

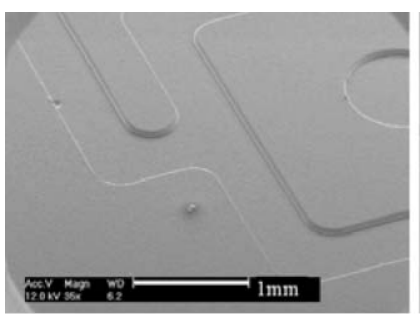

(a)

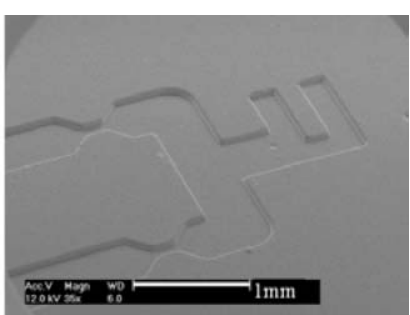

(b)

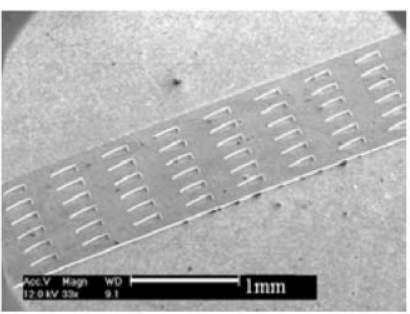

(c)

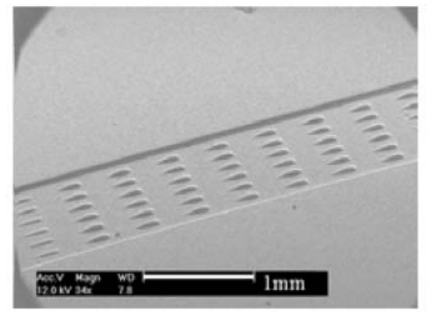

(c)
Fig. 4 Tilted SEM pictures of the electroplated nickel positive relief (cavities) in the fabricated mould inserts: (a) flow splitting microchannel; (b) F-shape mixing unit of the SLM; and (c) detection microfilter of $80 \mu \mathrm{m}$ gap.

electroplated nickel positive relief on the nickel substrate from the SEM pictures of Fig. 4.

\section{Fabrication of biochip}

As was discussed above, we applied the microinjection moulding process as a mass production method for making the microfluidic biochip in order to achieve cost-effectiveness. Two polymer substrates of the biochip were replicated by means of a conventional injection moulding machine (Allrounders $220 \mathrm{M}$, Arburg) with the fabricated mould base and mould inserts as shown schematically in Fig. 2(e). In this case, cyclic olefin copolymer (COC; Topas 5013S-04, Ticona) was chosen as the substrate material of the biochip after considering its great advantages over the other thermoplastic polymers in terms of physical and chemical properties and biocompatibility. ${ }^{13,14}$ Table 2 lists the detailed processing conditions of the microinjection moulding process applied in this study. It should be noted that the total cycle time of the present microinjection moulding process was less than $1 \mathrm{~min}$ to replicate the COC substrate. Fig. 5 shows representative titled SEM pictures of the replicated microstructures in the COC substrate.

The microfluidic biochip was finally made by thermal bonding of the two injection moulded COC substrates by means of a hot embossing machine after drilling holes $(0.8 \mathrm{~mm}$

Table 2 Processing condition of the microinjection moulding process

\begin{tabular}{ll}
\hline Processing condition & Value \\
\hline Injection melt temperature & $290{ }^{\circ} \mathrm{C}$ \\
Mould temperature & $110{ }^{\circ} \mathrm{C}$ \\
Injection pressure & $300 \mathrm{bar}$ \\
Injection filling time & $0.25 \mathrm{~s}$ \\
Packing pressure & $300 \mathrm{bar}$ \\
Packing time & $2 \mathrm{~s}$ \\
Cooling time & $30 \mathrm{~s}$ \\
\hline
\end{tabular}

Fig. 5 Tilted SEM pictures of the injection moulded microstructures in the COC substrates: (a) flow splitting microchannel; (b) F-shape mixing unit of the SLM; and (c) detection microfilter with $80 \mu \mathrm{m}$ gap.

diameter drill bit) for fluidic interconnection for inlets and outlets on the top side COC substrate, as depicted in Fig. 2(f). The thermal bonding process was carried out at a heating temperature of $123{ }^{\circ} \mathrm{C}$ and an applied pressure of $1.94 \mathrm{MPa}$ for a duration time of $10 \mathrm{~min}$. It should be noted here that thermal bonding of the present reaction microchamber requires special attention to prevent the collapse of the microchamber due to its large area. We could successfully form the reaction microchamber without the collapse via a holed pressure equalizing plate (HPEP) which includes holes of the same size and shape as the reaction microchambers. ${ }^{15}$ One may refer to the detailed bonding technique including the fabrication of the HPEP in ref. 15. During the bonding process, two replicated COC substrates were precisely aligned via the self-alignment microstructures, as indicated in Fig. 2(f). The final size of the individual biochip is $56 \times 44 \times 3.8 \mathrm{~mm}^{3}$. It should be noted that the total fabrication cycle time of each biochip (from the microinjection moulding to the thermal bonding) was just less than $20 \mathrm{~min}$ ( $1 \mathrm{~min}$ for the injection moulding, $3 \mathrm{~min}$ for the post processing of mechanical cutting and drilling of injection moulded COC substrates and $11 \mathrm{~min}$ for the thermal bonding). Fig. 6 shows the finally fabricated integrated microfluidic biochip with two blood typing units in which flow splitting microchannels, SLM's, reaction microchambers and detection microfilters are fully assembled.

\section{ABO blood typing}

\section{Experimental}

In this study, the red cell blood typing for $\mathrm{ABO}$ blood groups was performed by means of human whole bloods and the standard sera, Anti-A and Anti-B, in order to check the feasibility of the developed microfluidic biochip. In this regard, we prepared the biochip for the 2 blood agglutination tests via two test lines. It might be mentioned that the blood stream requires 2 times higher input flow rate than the sera streams 


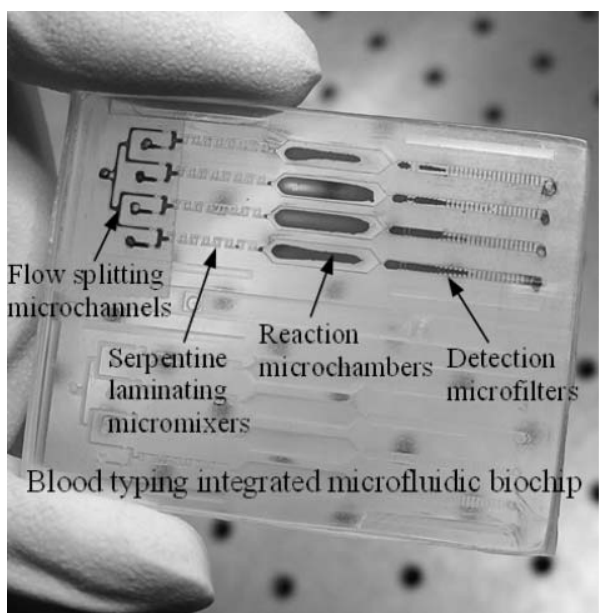

Fig. 6 Blood typing microfluidic biochip in which flow splitting microchannels, SLMs, reaction microchambers and detection microfilters are fully integrated. Red dyed water was injected into one blood typing unit of the biochip to enhance the observation.

since the blood stream splits into 2 sub-streams by the flow splitting microchannel. To flexibly handle two different flow rates of the blood and sera streams, two syringe pumps (kdScientific Inc., KDS200) were utilized. Fig. 7 shows the blood typing experimental setup. The experimental procedure of blood typing according to the operation process introduced above is as follows.

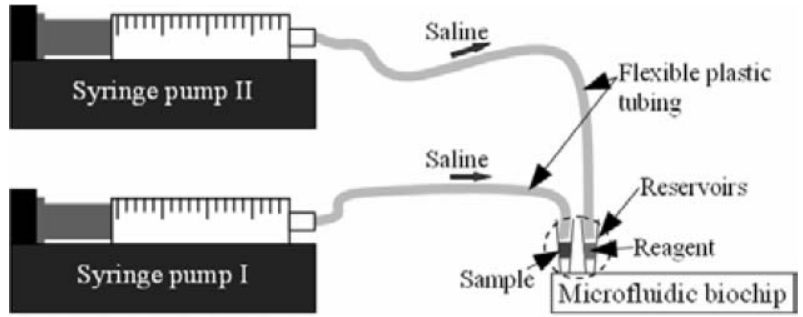

(a)

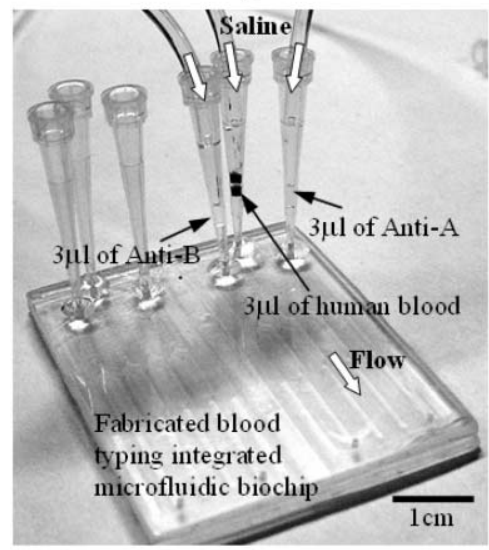

(b)

Fig. 7 Blood typing experimental setup with the developed microfluidic biochip: (a) schematic diagram of the configuration; and (b) microfluidic biochip installed in the blood typing experimental setup. Human whole blood and standard sera (Anti-A and Anti-B) were already loaded.
(1) First, three types of sample human blood (blood groups of $\mathrm{A}, \mathrm{B}$ and $\mathrm{AB}$ ) were withdrawn from three healthy young donors. (It might be mentioned that about $1 \mathrm{ml}$ of sample blood was withdrawn from each donor by means of a disposable syringe.) The sample whole bloods were mixed with the heparin in a 6:1 volume ratio to prevent coagulation.

(2) The test volume of $3 \mu \mathrm{l}$ of each whole blood was loaded into the blood inlet reservoir of each microfluidic biochip and also each $3 \mu \mathrm{l}$ of Anti-A and Anti-B (Murine IgM monoclonal, Serologicals Ltd.) was loaded into serum inlet reservoirs, as indicated in Fig. 7.

(3) The loaded sample whole blood and the standard sera were injected into the biochip by means of two syringe pumps with the help of saline as a working fluid through the tubing until the blood and sera flow into the reaction microchambers through the flow splitting microchannels and SLMs.

(4) The mixture of the whole blood and sera was kept for about $2 \mathrm{~min}$ as the reaction time of agglutination.

(5) The saline was then injected into the biochip only through the blood inlet (i.e., using only one syringe pump for blood injection) until the reacted or non-reacted mixtures of blood and sera pass through the detection microfilters and then flow out from the outlets.

(6) We observed the detection microfilters, whether agglutinated RBCs were filtered or not, with the naked eye and then we finally determined the blood group of the three sample bloods based on the filtering results.

In this study, we have also carried out the plate-type blood typing in order to confirm the blood typing results from the biochip. In the plate-type blood typing, each $20 \mu \mathrm{l}$ of the sample whole blood (i.e., a total of $40 \mu \mathrm{l}$ of the whole blood used) was first loaded at two test wells. Each $20 \mu$ of Anti-A and Anti-B was then loaded at the two test wells and they were manually mixed with the already loaded blood. After $3 \mathrm{~min}$ of agglutination time, we observed the agglutination results.

\section{Blood typing results}

To determine the proper operating flow rate, we carried out the blood typing experiments, changing the flow rate of the saline from the syringe pumps. At the high flow rate (e.g., $50 \mu 1 \mathrm{~min}^{-1}$ ), it was found that the agglutinated RBCs were mechanically broken up while passing the detection microfilter due to the high shear stress induced by the flow. According to the parametric study on the flow rate, the optimal flow rate was found to be $30 \mu \mathrm{min}^{-1}$ for the $\mathrm{ABO}$ blood typing in the present study. For this condition, Re becomes 2.0, based on the plasma specific gravity and viscosity of 1.03 and $1.2 \mathrm{cP}$, respectively. ${ }^{16}$ The SLM requires about $6 \mathrm{~mm}$ of a characteristic required length to mix the two fluid streams around $R e$ of 2.0 according to our previous result. ${ }^{9}$ The present design of the SLM has $10 \mathrm{~mm}$ of total length and therefore, under this condition, we could expect the efficient mixing of the blood and serum by means of the present SLM.

Fig. 8 shows the successful blood typing results of the three sample bloods. Insets in Fig. 8 show microscope views of the successfully filtered agglutinated RBCs and air entrapments in the detection microfilters (and the reaction microchambers) 


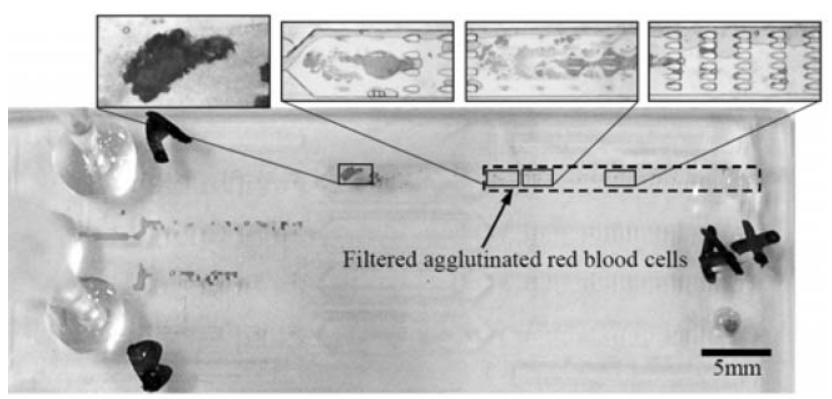

(a)

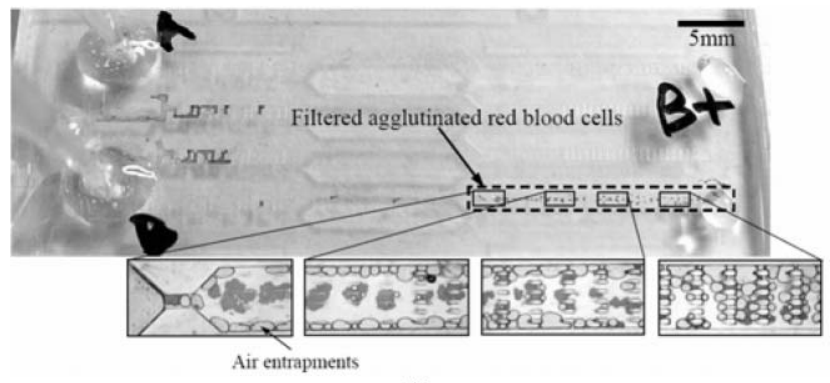

(b)

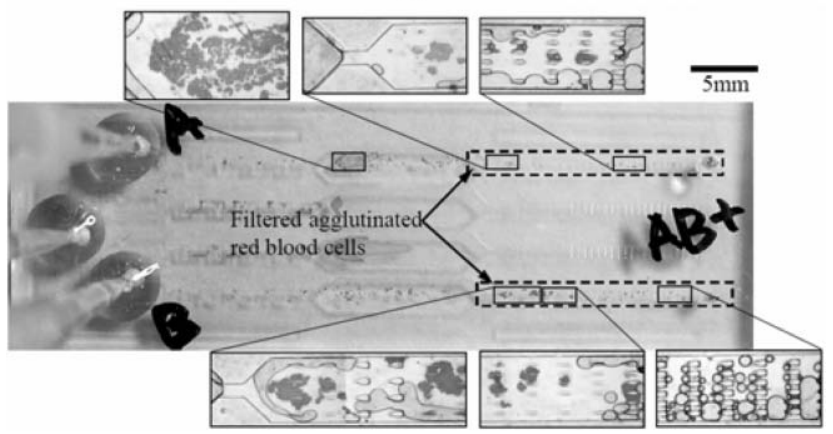

(c)

Fig. 8 ABO blood typing results of the three sample bloods by means of the blood typing integrated microfluidic biochip developed in this study: (a) blood group A; (b) blood group B; and (c) blood group AB. Insets in the figures are enlarged microscope views of the filtered agglutinated red cells.

after flushing out. It should be noted that one can easily observe the filtered agglutinated RBCs in the detection microfilters by the naked eye from the blood typing results in Fig. 8. Therefore, one is able to simply and precisely determine the blood group of the sample bloods with the blood typing integrated microfluidic biochip developed in this study. We have carried out more than three blood typing tests per each blood sample and confirmed all the successful identification of agglutination of blood samples. Also, we have found that the blood typing results of the biochip were in exact accordance with the results of the plate-type blood typing method performed in this study (see electronic supplementary information $\uparrow$ for blood typing results of the plate-type method).

It may be mentioned that the size of the agglutinated red cells of blood group A is generally so large that they could not pass the relatively narrow microchannel in front of the microfilter, thereby frequently remaining in the reaction microchamber, as shown in Fig. 8 (a) and (c). This might be explained by the high reaction activity of the agglutinogen of blood group A with the corresponding agglutinin.

\section{Conclusions}

In this study, we have designed, fabricated and characterized a low-cost, disposable, integrated microfluidic biochip for blood typing in which flow splitting microchannels, serpentine laminating chaotic micromixers, reaction microchambers and detection microfilters are fully incorporated. By means of the present flow splitting microchannels, it was possible to carry out 2 or 4 blood agglutination tests in parallel. For efficient mixing of red blood cells (RBCs) and serum, the serpentine laminating micromixer was introduced into the biochip. The fully mixed RBCs and serum were kept in the large area reaction microchamber in the biochip. The agglutinated RBCs were successfully filtered by the gradually decreasing detection microfilter, designed in this study, and thereby one can easily and precisely determine the blood group of sample bloods by means of the developed biochip.

To achieve cost effectiveness of the biochip, we have manufactured it by the microinjection moulding of $\mathrm{COC}$ (cyclic olefin copolymer) and thermal bonding of two injection moulded COC substrates with a total fabrication time of less than $20 \mathrm{~min}$. The mould inserts for the microinjection moulding process were fabricated by SU-8 photolithography and subsequent nickel electroplating.

For the feasibility test of the developed biochip, we have demonstrated successful ABO blood typing results with the fabricated biochip. Three kinds of human blood groups, A, B and $A B$, were successfully determined with $3 \mu$ of the whole blood within a test time of 3 min by means of the fabricated biochip.

Based on the results of the present work, we want to simply mention the advantages of the present biochip in terms of cost, rapidity, easiness and reliability, although it might be too early to discuss this last item. The present biochip can reduce the reagent cost since the biochip requires a smaller test volume (about $3 \mu \mathrm{l}$ ) than the previous methods (more than $20 \mu \mathrm{l}$ ). The fabrication cost of the biochip may be also less than the gel card, automated system and miniaturized system, since we applied a mass fabrication method to the fabrication of the biochip. The microfluidic channel of the biochip increases surface to volume ratio so that it could enhance the reaction of agglutinogens on RBCs and agglutinins in serum, thereby resulting in the fast testing time. Because of the compact size of the biochip, it is easy to handle. The multi-step microfilter of the biochip increases chances to filter out agglutinated RBCs and, therefore, it can increase the reliability of the biochip. Also, one can preserve the blood typing test result performed with the biochip.

This integrated microfluidic biochip developed in this study may form the basis of development of microfluidic devices for automated diagnosis, suitable for the level of hospitals in function and capacity. In addition to that, the proposed biochip could be applied to clinical examinations based on agglutination reactions in antigen-antibody reactions, not to mention the blood typing. 


\section{Acknowledgements}

The authors would like to thank to the Korean Ministry of Science and Technology for their partial financial support via a research grant for the National Research Laboratory Program (2000-N-NL-01-C-148) and also thank to the Korean Ministry of Commerce, Industry and Energy for partial financial supports via a research project of Micro-Injection/ Compression Moulding Technology for Polymer-based Microparts and a research project of Development of NextGeneration New Technology (Development of Intelligent Robot Technologies for Laboratory Medicine by Applying Biotechnology). The authors also thank to Professor DongWoo Cho, Mr. Seung Jae Lee and Mr. Bong-Kee Lee, in the department of mechanical engineering, POSTECH, and Professor In Hwan Lee in the department of mechanical engineering, Chungbuk National University is also thanked for very helpful technical discussions.

\section{References}

1 K. S. Han, M. H. Park and S. I. Kim, Transfusion Medicine, Korea Medical Book Publisher, 1999 (in Korean).

2 V. Migeot, I. Ingrand, L. R. Salmi and P. Ingrand, 'Reliability of bedside ABO testing before transfusion', Transfusion, 2002, 42, $1348-1355$.

3 M. M. Langston, J. L. Procter, K. M. Cipolone and D. F. Stroncek, 'Evaluation of the gel system for ABO grouping and D typing', Transfusion, 1999, 39, 300-305.

$4 \mathrm{http}: / / \mathrm{www}$. diamed.com.

5 F. Morelati, N. Revelli, L. M. Maffei, M. Poretti, C. Santoro, A. Parravicini, P. Rebulla, R. Cole and G. Sirchia, 'Evaluation of a new automated instrument for pretransfusion testing', Transfusion, 1998, 38, 959-965.

$6 \mathrm{http} / / /$ www.olympusamerica.com/dsg_section/dsg_bloodbank.asp.

7 S.-J. Lee, H.-W. Kang, Y. Kim, G.-W. Lee, G. Lim and D.-W. Cho, 'Development of a micro-blood-typing system using micro-stereolithography', Sens. Mater., 2005, 17, 113-123.

8 D. S. Kim, I. H. Lee, T. H. Kwon and D.-W. Cho, 'A barrier embedded Kenics micromixer', J. Micromech. Microeng., 2004, 14, 1294-1301.

9 D. S. Kim, S. H. Lee, T. H. Kwon and C. H. Ahn, 'A serpentine laminating micromixer combining splitting/recombination and advection', Lab Chip, 2005, 5, 739-747.

10 D. S. Kim, K.-C. Lee, T. H. Kwon and S. S. Lee, 'Micro-channel filling flow considering surface tension effect', J. Micromech. Microeng., 2002, 12, 236-246.

11 D. C. Duffy, J. C. McDonald, O. J. A. Schueller and G. M. Whitesides, 'Rapid prototyping of microfluidic systems in poly(dimethylsiloxane)', Anal. Chem., 1998, 70, 4974- 4984.

12 S. H. Lee, J. Han, D. S. Kim, T. H. Kwon, C. J. Hwang, Y. M. Heo and C. H. Ahn, A high precision self-assembly technique for multilayer polymer lab-on-a-chip, in Proceedings of Micro Total Analysis Systems 2004 ( $\mu$ TAS2004), Malmö, Sweden, September 2004, vol. 2, pp. 413-415.

13 C. H. Ahn, J.-W. Choi, G. Beaucage, J. H. Nevin, J.-B. Lee, A. Puntambekar and J. Y. Lee, 'Disposable smart lab on a chip for point-of-care clinical diagnostics', Proc. IEEE, 2004, 92, 154-173.

$14 \mathrm{http} / / / \mathrm{www}$. ticona-norden.com/home/portfolio/topas.htm.

15 D. S. Kim, H. S. Lee, T. H. Kwon and C. H. Ahn, 'A collapse-free thermal bonding technique for plastic microfluidic systems with large area microchamber', in Proceedings of Micro Total Analysis Systems 2005 ( $\mu$ TAS2005), Boston, USA, October 2005, pp. 684 686; D. S. Kim, H. S. Lee, J. Han, S. H. Lee, C. H. Ahn and T. H. Kwon, unpublished work.

16 Y. C. Fung, Biomechanics: Mechanical Properties of Living Tissues, Springer-Verlag, New York, 1981. 